\title{
MICROSCOPIC ANALYSIS OF CORE DEFORMATION FOR NUCLEI IN THE PROTON-RICH Xe-Nd REGION
}

\author{
D. BRAUNSCHWEIG and K.T. HECHT \\ Physics Department, University of Michigan, Ann Arbor, MI 48109, USA
}

Received 9 March 1978

Revised manuscript received 25 May 1978

A shell model study in terms of a severely truncated pseudo-SU $\mathrm{U}_{3}$ basis has been made of typical rotational-limit nuclei in the proton-rich $\mathrm{Xe}-\mathrm{Nd}$ region. The competition between prolate and oblate shapes and $\mathrm{E} 2$ properties have been studied.

In light nuclei the rotational features of nuclear spectra can be understood in a shell model framework in terms of Elliott's $\mathrm{SU}_{3}$ coupling scheme. In a heavy deformed nucleus, however, the conventional $\mathrm{SU}_{3}$ model is not applicable, and the size of a realistic shell model basis for such a nucleus in the $j-j$ coupling scheme would be so enormous that reasonable calculations are impossible. The pseudo-SU $\mathrm{SU}_{3}$ model $[1,2]$ on the other hand may lead to the severe but realistic truncation of the shell model space needed for microscopic studies of deformed nuclei in more challenging regions of the periodic table. Initial calculations for nuclei in the rare earth region in terms of the pseudo$\mathrm{SU}_{3}$ scheme $[1,3]$, although promising, have been restricted to model studies based on configurations of natural parity orbitals of identical nucleons and thus focus attention only on those nuclear properties which are governed mainly by that part of the full nucleon configuration, e.g. the $\left(g_{7 / 2} d_{5 / 2} d_{3 / 3} s_{1 / 2}\right)^{n}$ proton configuration for odd- $Z$ rare earth nuclei. To establish the validity of the pseudo-SU $\mathrm{S}_{3}$ model, more detailed shell model calculations in a full neutrontproton configuration have to be performed. The recent development of a very general computer code [4] which calculates reduced matrix elements for arbitrary one- and two-body $\mathrm{SU}_{3}$-tensor operators provides us with the necessary tools to perform more challenging calculations in de-

\footnotetext{
Supported by the U.S. National Science Foundation.
}

formed nuclei. The highly neutron deficient nuclei in the $\mathrm{Xe}-\mathrm{Nd}$ region, a region of large deformations [5], have been chosen as the most promising area for an initial study, since the valence neutrons and protons are filling the same shell model orbits, the $\mathrm{g}_{7 / 2} \mathrm{~d}_{5 / 2} \mathrm{~d}_{3 / 3} \mathrm{~s}_{1 / 2}$ orbits which are treated as a pseudo $\widetilde{\mathrm{f}}_{7 / 2} \mathrm{f}_{5 / 2} \widetilde{\mathrm{p}}_{3 / 2} \widetilde{\mathrm{p}}_{1 / 2}$ shell, and the unnatural parity intruder orbit $h_{11 / 2}$. A fult shell model description will have to take into account the interplay between the filling of the $h_{11 / 2}$ orbit and the $g_{7 / 2} d_{5 / 2} d_{3 / 2} s_{1 / 2}$ part of the shell model space, truncated in terms of pseudo-SU $\mathrm{S}_{3}$ symmetry. Since the deformation and quadrupole collective properties of such nuclei are correlated mainly with the $\mathrm{g}_{7 / 2} \mathrm{~d}_{5 / 2} \mathrm{~d}_{3 / 2} \mathrm{~s}_{1 / 2}$ part of the configuration, an initial model study of even-even nuclei based on this part of the shell model configuration has been undertaken. Our aim is hence not to fit spectra of specific nuclei, but to study the goodness of pseudo- $\mathrm{SU}_{3}$ symmetry in large configurations of both $\tilde{\mathrm{fp}}$ protons and neutrons by comparisons with a typical "rotational limit" nucleus of this region. In particular, we would require the pseudo-SU model to give $B \mathrm{E} 2$ values in the observed range with reasonable effective charges. The pseudo- $\mathrm{SU}_{3}$ model can also shed some light on the long-standing question of prolate-oblate competition [6] in this region.

The many-particle basis states for the $g_{7 / 2} \mathrm{~d}_{5 / 2} \mathrm{~d}_{3 / 2}$ $\mathrm{s}_{1 / 2}$ or $\widetilde{\mathrm{fp}}$ configurations are denoted by

$\left|[\tilde{f}] \alpha \beta(\tilde{\lambda \mu}) \tilde{K}(\tilde{L} \tilde{S}) J M_{J} T M_{T}\right\rangle$, 
where $[\tilde{f}]$ and $(\tilde{\lambda \mu})$ give the $\mathrm{U}_{10}$ and $\mathrm{SU}_{3}$ symmetries. (A detailed description of the quantum numbers is given in ref. [1].) Nucleons in the $h_{11 / 2}$ orbit are assumed to be coupled to an inert seniority zero state. Since surface delta and delta-like effective interactions [7] have been shown to be good effective interactions in highly truncated shell model spaces, the simple surface delta interaction has been chosen as the effective two-body interaction. The effective hamiltonian was taken to be

$H=A \sum_{i=1}^{n} \tilde{l}_{i} \cdot \tilde{s}_{i}+B \sum_{i=1}^{n} \tilde{l}_{i}^{2}-G H_{\mathrm{SDI}}$

where $A$ was adjusted so that the splitting between $\mathrm{g}_{7 / 2}$ and $\mathrm{d}_{5 / 2}$ levels is $0.875 \mathrm{MeV}$, while $B$ was chosen so that the splitting between the centers of gravity of the $\mathrm{s}_{1 / 2} \mathrm{~d}_{3 / 2}$ and $\mathrm{d}_{5 / 2} \mathrm{~g}_{7 / 2}$ doublets is $2.25 \mathrm{MeV}$. The strength of $G$ was chosen so that the diagonalization of $G H_{\mathrm{SDI}}$ in the 2-particle space gives a depression of 1 $\mathrm{MeV}$ for the favoured $J=0$ state, corresponding to a reasonable pairing strength for nuclei in the $A=120$ 140 range.

In strongly deformed nuclei the truncation of the shell model space in terms of pseudo $\mathrm{SU}_{3}$ symmetry is meaningful if only pseudo $\mathrm{SU}_{3}$ representations near the leading representations (highest possible $2 \tilde{\lambda}+\tilde{\mu}$ for prolate, highest $2 \tilde{\mu}+\tilde{\lambda}$ for oblate shapes) are needed for low-lying states, thus assuring maximum possible deformation. Although the experimental evidence [5] for neutron deficient nuclei with $Z>54$ clearly favors prolate deformation, the history of the theoretical predictions [6] for the shapes of nuclei in this region seems to indicate a possible strong competition between oblate and prolate shapes. The first step in the truncation procedure must thus involve a comparison of the unperturbed positions (diagonal matrix elements) of the most prolate (highest $2 \widetilde{\lambda}+\widetilde{\mu}$ ) and oblate (highest $2 \tilde{\mu}+\tilde{\lambda})$ pseudo-SU $\mathrm{S}_{3}$ representations. The case of 20 natural parity nucleons (the half-full fp shell) is of special interest since all possible pseudo $\mathrm{SU}_{3}$ representations in this case occur in conjugate $(\tilde{\lambda \mu})(\tilde{\mu} \lambda)$ pairs with equal values for their Casimir invariants, hence identical eigenvalues for a pseudo $\widetilde{Q} \cdot \widetilde{Q}$ interaction, and effectively identical eigenvalues for a real $Q \cdot Q$ interaction. Fig. 1 shows nevertheless that the effective hamiltonian (2) clearly favors prolate shapes in nuclei with large numbers of protons and neutron holes. For

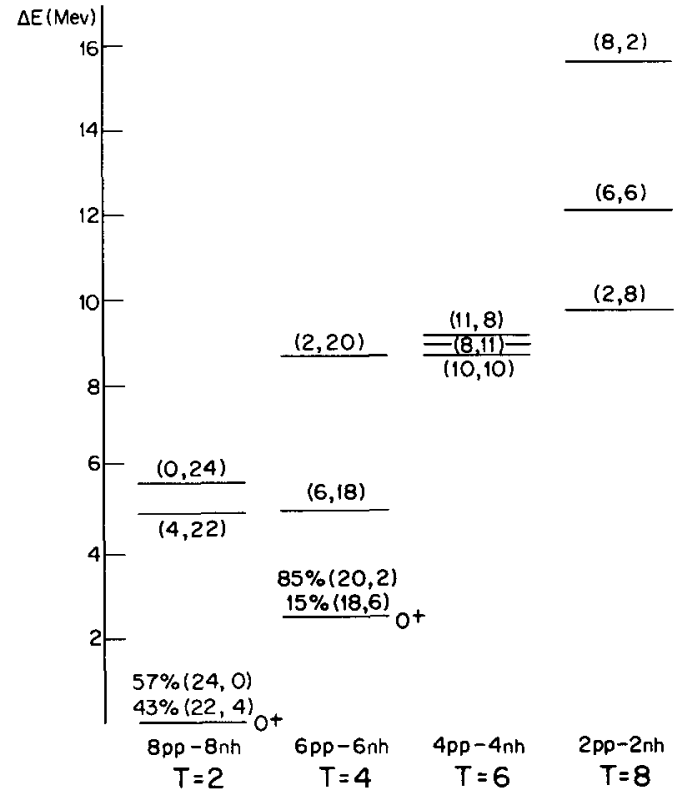

Fig. 1. Competition between prolate and oblate shapes in the $(\widetilde{\text { pf }})^{20}$ configuration. For the low-lying states, the percentages of the major $(\tilde{\lambda \mu})$ components are indicated. The high-lying levels indicate the unperturbed positions (diagonal matrix elements) of the $(\tilde{\mu} \tilde{\lambda})$ states of opposite shape.

nuclei with 8 natural parity protons and 8 natural parity neutron holes, e.g., there are two competing representations with $2 \vec{\lambda}+\tilde{\mu}=48,(\tilde{\lambda \mu})=(24,0)$ and $(22,4)$. These are the major components of the lowest lying state while the unperturbed position of the $(4,22)$ and $(0,24)$ states lie at an excitation energy of about $5 \mathrm{MeV}$ (and would in fact be pushed to even higher energy by prolate excitations of intermediate $2 \tilde{\lambda}+\tilde{\mu}$ ). Since near-rotational nuclei in the neutron-deficient Ba region can be expected to have around 6 proton particles and 8 neutron holes in the natural parity space, we predict a prolate shape and use a truncation scheme in terms of large $2 \widetilde{\lambda}+\widetilde{\mu}$ for this region. It is interesting to note, however, that oblate shapes seem to win out for nuclei with very large neutron excess and that the shape competition is most pronounced for the $4 \mathrm{p}-4 \mathrm{~h}$ case. As we traverse the $A=132$ isotones in the direction of decreasing neutron number we might therefore expect the deformation to become more and more prolate with a possible change of shape around ${ }^{132} \mathrm{Xe}$. (However, our truncation scheme is invalid in the neighborhood of closed shell nuclei.)

To test the validity of the pseudo-SU $\mathrm{S}_{3}$ model the 
effective hamiltonian (2) was diagonalized in a truncated high- $2 \tilde{\lambda}+\tilde{\mu}(\tilde{\mathrm{fp}})$ shell model basis for several nuclei of prolate shape. The case of 6 proton particles and 8 neutron holes leads to a pure $K=0$ ground state rotational band since the leading $(2 \tilde{\lambda}+\tilde{\mu})$-space consists of the single representation $(\tilde{\lambda \mu})=(24,0)$. In a basis including all states with $2 \tilde{\lambda}+\tilde{\mu} \geqslant 45$ the $2^{+}$member of the ground state rotational band, e.g., has eigenvector

$$
\begin{aligned}
& 0.950|(24,0)\rangle+0.210|(22,1)\rangle-0.222\left|(21,3)_{1}\right\rangle \\
& -0.064\left|(21,3)_{2}\right\rangle-0.019|(20,5)\rangle,
\end{aligned}
$$

where the basis vectors of eq. (1) are abbreviated by $|(\tilde{\lambda \mu})\rangle$; (note that $(\tilde{\lambda \mu})=(21,3)$ has a double occurrence for states with $\left.[\tilde{f}]=\left[4^{3} 2^{3}\right] \tilde{s}=0, T=3\right)$. Inclusion of states with $2 \widetilde{\lambda}+\tilde{\mu}=42$ would enlarge the basis to one of dimensionality 52 for $J=2$; but examination of our $H_{\text {effective }}$ shows that admixtures of the additional $(\tilde{\lambda \mu})$ components into the ground state rotational band would each be much less that $1 \%$ so that the remarkable purity of the $(\tilde{\lambda \mu})=(24,0)$ representation can be expected to be preserved. This purity is not a trivial consequence of the choice of effective interaction, since the hamiltonian of eq. (2) can lead to large $(\tilde{\lambda \mu})$ mixing in nearly spherical nuclei. The hamiltonian of eq. (2) (with coefficients identical to those chosen here) has been used by Strottman [8] in his study of the pseudo $(\tilde{\mathrm{fp}})$ shell configurations $(\tilde{\mathrm{fp}})^{3}$ and $(\tilde{\mathrm{fp}})^{4}$ of identical nucleons. For these configurations, which should be good approximations for the semi-magic nuclei ${ }_{53}^{135} \mathrm{I}_{82}$ and ${ }_{54}^{136} \mathrm{Xe}_{82}$, he has shown that the hamiltonian (2) leads to a very large mixing of pseudo-SU $\mathrm{SU}_{3}$ representations; $(\tilde{\lambda \mu})$ are not even approximately good quantum numbers in these non-collective nuclei. As expected, the purity of the pseudo$\mathrm{SU}_{3}$ symmetry, along with the collectivity, is thus a function of the number of particles and holes. Unfortunately, the huge pseudo-SU 3 shell model diagonalizations which would be needed to make a more detailed study of the transition, as well as a more complete study of the pseudo $\mathrm{SU}_{3}$ purity, are beyond our present computer capacity.

The $6 p-6$ h case included in fig. 1 was examined in more detail. The ground state rotational band is an almost pure $K=0$ band which is a mixture of the pseudo$\mathrm{SU}_{3}$ representations $(20,2)$ and $(18,6)$ with $2 \tilde{\lambda}+\tilde{\mu}=42$. Fig. 2 shows that the admixture of the most important

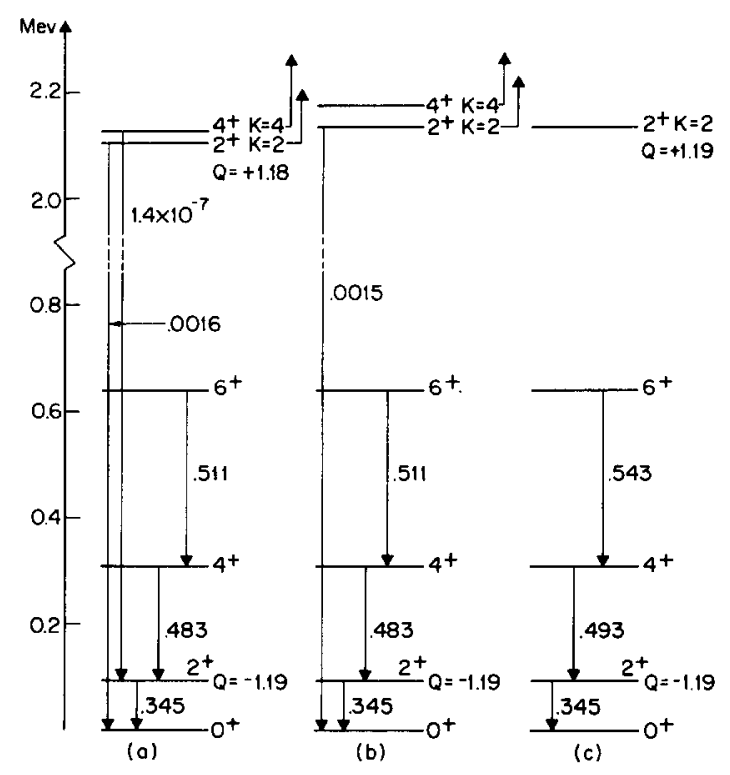

Fig. 2. Spectrum for the 6 proton-particle -6 neutron-hole $T=4(\widetilde{\mathrm{pf}})^{20}$ configuration. (a) Pseudo- $\mathrm{SU}_{3}$ spectrum, $(20,2)$ $+(18,6)$ truncation. (b) $(20,2)+(18,6)+(15,9)^{2}$ truncation. (c) Comparison "rotational-limit" nucleus. The numbers give $B E 2$-values $\left(10^{-48} \mathrm{~cm}^{4}\right)$ and a quadrupole moments $\left(10^{-24}\right.$ $\mathrm{cm}^{2}$ ) with effective charges of 0.5 for (a) and (b), $\beta=0.26$ for (c).

representations with $2 \tilde{\lambda}+\tilde{\mu}=39$, two separate representations with $(\tilde{\lambda \mu})=(15,9)$, has essentially no effect on either the rotational spacing or the $B \mathrm{E} 2$ values of this spectrum. The $2^{+}$member of the $K=2$ band is a mixture of $47 \%(20,2)$ and $53 \%(18,6) K=2$ wave functions with an admixture of only $0.1 \%(15,9)$. The pseudo$\mathrm{SU}_{3}$ spectra are compared with that of a symmetric rotator with an intrinsic quadrupole moment of $Q_{0}=$ $4.165 \mathrm{~b}$, corresponding to a Bohr deformation parameter $\beta=0.26$, a reasonable $\beta$-value for a typical "rotational limit" nucleus of the neutron deficient Ba region. The quadrupole moments and $B E 2$ values shown in columns (a) and (b) have been calculated with effective proton and neutron charges of $e_{\pi}=e(1+0.5)$ and $e_{v}=(0.5)$. The value of 0.5 is a very reasonable one for an effective charge since it must account for core deformation effects in a shell model description in terms of 20 active valence nucleons in a nucleus with $A \approx 130$. (Since a 6 proton -6 neutron hole $(\tilde{\mathrm{fp}})$ configuration can be expected to be coupled predominantly to a 2 proton-4 neutron hole $\mathrm{h}_{11 / 2}$ configuration, the above might describe the $\widetilde{\mathrm{fp}}$ spectrum of ${ }^{130} \mathrm{Ce}$.) 
Table 1

Pseudo- $\mathrm{SU}_{3}$ tensor decomposition of the quadrupole operator.

\begin{tabular}{|c|c|c|c|c|c|}
\hline $\begin{array}{l}\text { Pseudo } \\
\text { oscillator } \\
\text { shell }\end{array}$ & $(\tilde{\lambda \mu})$ & $\tilde{K}$ & $\tilde{L}$ & $\tilde{S}$ & $C_{(\tilde{\lambda \mu})} \widetilde{K} \widetilde{L} \widetilde{S}$ \\
\hline \multirow[t]{7}{*}{$\widetilde{N}=2$} & $\left(\begin{array}{ll}1 & 1\end{array}\right)$ & 1 & 1 & 1 & -0.1993 \\
\hline & $\left(\begin{array}{ll}1 & 1\end{array}\right)$ & 1 & 2 & 0 & 4.8720 \\
\hline & $\left(\begin{array}{ll}2 & 2\end{array}\right)$ & 0 & 2 & 0 & -0.0385 \\
\hline & $\left(\begin{array}{ll}2 & 2\end{array}\right)$ & 0 & 2 & 1 & 0.2986 \\
\hline & $\left(\begin{array}{ll}2 & 2\end{array}\right)$ & 2 & 2 & 0 & 0.0789 \\
\hline & $\left(\begin{array}{ll}2 & 2\end{array}\right)$ & 2 & 2 & 1 & 0.1457 \\
\hline & $\left(\begin{array}{ll}2 & 2\end{array}\right)$ & 2 & 3 & 1 & 0.1421 \\
\hline \multirow[t]{11}{*}{$\tilde{N}=3$} & $\left(\begin{array}{ll}1 & 1\end{array}\right)$ & 1 & 1 & 1 & -0.2902 \\
\hline & $\left(\begin{array}{ll}1 & 1\end{array}\right)$ & 1 & 2 & 0 & 8.1885 \\
\hline & $\left(\begin{array}{ll}2 & 2\end{array}\right)$ & 0 & 2 & 0 & -0.0751 \\
\hline & $\left(\begin{array}{ll}2 & 2\end{array}\right)$ & 0 & 2 & 1 & 0.5715 \\
\hline & $\left(\begin{array}{ll}2 & 2\end{array}\right)$ & 2 & 2 & 0 & 0.1540 \\
\hline & $\left(\begin{array}{ll}2 & 2\end{array}\right)$ & 2 & 2 & 1 & 0.2788 \\
\hline & $\left(\begin{array}{ll}2 & 2\end{array}\right)$ & 2 & 3 & 1 & 0.2638 \\
\hline & $\left(\begin{array}{ll}3 & 3\end{array}\right)$ & 1 & 1 & 1 & -0.0071 \\
\hline & (3 3) & 1 & 2 & 0 & 0.1589 \\
\hline & (3 3) & 1 & 3 & 1 & -0.0170 \\
\hline & $\left(\begin{array}{ll}3 & 3\end{array}\right)$ & 3 & 3 & 1 & 0.0249 \\
\hline \multirow[t]{16}{*}{$\tilde{N}=4$} & $\left(\begin{array}{ll}1 & 1\end{array}\right)$ & 1 & 1 & 1 & -0.3827 \\
\hline & (1 1 ) & 1 & 2 & 0 & 12.2228 \\
\hline & $\left(\begin{array}{ll}2 & 2\end{array}\right)$ & 0 & 2 & 0 & -0.1156 \\
\hline & $\left(\begin{array}{ll}2 & 2\end{array}\right)$ & 0 & 2 & 1 & 0.8663 \\
\hline & $\left(\begin{array}{ll}2 & 2\end{array}\right)$ & 2 & 2 & 0 & 0.2369 \\
\hline & $\left(\begin{array}{ll}2 & 2\end{array}\right)$ & 2 & 2 & 1 & 0.4227 \\
\hline & $\left(\begin{array}{ll}2 & 2\end{array}\right)$ & 2 & 3 & 1 & 0.3922 \\
\hline & $\left(\begin{array}{ll}3 & 3\end{array}\right)$ & 1 & 1 & 1 & -0.0142 \\
\hline & (3 3 ) & 1 & 2 & 0 & 0.3269 \\
\hline & (3 3 ) & 1 & 3 & 1 & -0.0348 \\
\hline & $\left(\begin{array}{ll}3 & 3\end{array}\right)$ & 3 & 3 & 1 & 0.0508 \\
\hline & $\left(\begin{array}{ll}4 & 4\end{array}\right)$ & 0 & 2 & 0 & -0.0069 \\
\hline & $\left(\begin{array}{ll}4 & 4\end{array}\right)$ & 0 & 2 & 1 & 0.0801 \\
\hline & $\left(\begin{array}{ll}4 & 4\end{array}\right)$ & 2 & 2 & 0 & 0.0133 \\
\hline & $\left(\begin{array}{ll}4 & 4\end{array}\right)$ & 2 & 2 & 1 & 0.0418 \\
\hline & $\left(\begin{array}{ll}4 & 4\end{array}\right)$ & 2 & 3 & 1 & 0.0426 \\
\hline
\end{tabular}

To calculate the quadrupole moments and the $B E 2$ values the quadrupole operator has been decomposed into pseudo- $\mathrm{SU}_{3}$ tensors [1]. The result is:

$$
\begin{aligned}
& Q_{\mathrm{op}}=\sum_{i=1}^{n}\left[\frac{16 \pi}{5}\right]^{1 / 2}\left\{e_{\pi}\left(\frac{1}{2}-t_{z i}\right)+e_{v}\left(\frac{1}{2}+t_{z i}\right)\right\} \\
& \times r_{i}^{2} Y_{20}\left(\theta_{i}, \phi_{i}\right)=\sqrt{2} \sum_{(\tilde{\lambda \mu}) \widetilde{K} \widetilde{L} \widetilde{S} T}\left(e_{v}+(-1)^{T} e_{\pi}\right) \\
& \times C_{(\tilde{\lambda \mu}) \tilde{K} \tilde{L} \tilde{S}}\left[a_{(\tilde{\eta} 0)}^{+} \times a_{(0 \tilde{\eta})}\right]_{M_{T}=0, M_{J}=0}^{(\tilde{\lambda \mu}) \tilde{K} \tilde{L} \tilde{S} T, J=2} .
\end{aligned}
$$

A tabulation of the coefficients $C_{(\tilde{\lambda \mu}) \tilde{K} \tilde{L} \tilde{S}}$ for the $\tilde{N}=2, \widetilde{N}=3$, and $\widetilde{N}=4$ shells is included in table 1 . (We note that the $(\tilde{N \mu})=(11)$ tensor predominates in all shells, so that the real $Q$ operator is effectively identical with the $Q$ operator of the pseudo space, a property to be expected from any operator whose matrix elements in $j-j$ coupling are independent of l.) The electromagnetic transition probabilities from state $I_{1}$ to state $I_{2}$ are given by

$$
\begin{aligned}
& B\left(\mathrm{E} 2 ; I_{1} \rightarrow I_{2}\right)=\left[\left(2 I_{2}+1\right) /\left(2 I_{1}+1\right)\right] \\
& \times\left\langle I_{2} \|[5 / 16 \pi]^{1 / 2} Q_{\mathrm{op}}|| I_{1}\right\rangle^{2},
\end{aligned}
$$

in terms of reduced matrix elements defined according to the conventions of ref. [1]. The numbers quoted in fig. 2 have used oscillator values for the matrix elements of $r^{2}$, with an oscillator length parameter $\hbar / m \omega_{0}$, with $\hbar \omega_{0}=\left[41 /(140)^{1 / 3}\right] \mathrm{MeV}$. It is worth noting that the value of the quadrupole moment for the $2^{+}$states of the $K=0$ and $K=2$ bands are almost equal in magnitude but opposite in sign in agreement with the predictions of the rotational model, an illustration of the purity of the $K$ values emerging from the pseudo-SU $\mathrm{SU}_{3}$ energy diagonalization.

We conclude that the truncation of the normal parity part of the shell model space in terms of pseudo-SU $\mathrm{SU}_{3}$ symmetry provides a reasonable microscopic description in strongly deformed nuclei, which is well suited to a more detailed analysis of nuclei in the neutron deficient $B$ a region.

[1] R.D. Ratna Raju, J.P. Draayer and K.T. Hecht, Nucl. Phys. A202 (1973) 433.

[2] A. Arima, M. Harvey and K. Shimizu, Phys. Lett. 30B (1969) 517.

[3] R.D. Ratna Raju, S. Reddy and V.K.B. Kota, J. Phys. G (1976) L29.

[4] D. Braunschweig, Comput. Phys. Commun., to be published.

[5] J.R. Leigh et al., Nucl. Phys. A213 (1973) 1;

K. Nakai et al., Phys. Lett. 44B (1973) 443;

J. Conrad et al., Nucl. Phys. A234 (1974) 157;

H. Kusakari et al., Nucl. Phys. A242 (1975) 13.

[6] K. Kumar and M. Baranger, Phys. Rev. Lett. 12 (1964) 73; J.A. Arseniev, A. Sobiczewski and V.G. Soloviev, Nucl. Phys. A126 (1969) 15;

I. Ragnarsson and S.G. Nilsson, Colloq. on Intermediate nuclei (Institut de Physique Nucleaire d'Orsay) IN 2 P3 p. 112 (1971);

J. Meyer-Ter-Vehn, Nucl. Phys. A249 (1975) 111, 165.

[7] J.P. Schiffer, in: The two-body force in nuclei, eds. S.M. Austin and G.M. Crowley (Plenum, New York, London, (1972).

[8] D. Strottman, Nucl. Phys. A279 (1977) 45. 